Cite this: J. Mater. Chem. C, 2013, 1, 351

Received 24th August 2012

Accepted 15th October 2012

DOI: $10.1039 / c 2 t c 00048 b$

www.rsc.org/MaterialsC

\section{Electrospinning of a blend of a liquid crystalline polymer with poly(ethylene oxide): Vectran nanofiber mats and their mechanical properties}

\author{
T. Medeiros Araujo, ${ }^{a}$ S. Sinha-Ray, ${ }^{b}$ A. Pegoretti ${ }^{a}$ and A. L. Yarin ${ }^{\star b}$ \\ Vectran ${ }^{\circledR}$ is a liquid crystalline polymer (LCP) with remarkable specific properties in its commercial \\ microfiber form. Even though it has been widely studied in the last few decades, there have been no \\ reports in the literature on Vectran nanofibers production. Due to the insufficient spinnability of \\ Vectran, a "host-guest" method with poly(ethylene oxide) (PEO) as a host polymer is used in the \\ present work to produce continuous and uniform nanofibers of Vectran-PEO blends. Subsequently, a \\ heat treatment is applied and optimized to remove PEO and convert the amorphous Vectran-PEO \\ nanofibers into more ordered and mechanically improved pure Vectran nanofibers. The conclusions are \\ supported by scanning electron microscopy, thermal analysis, selected area electron diffraction (SAED) \\ patterns and mechanical characterization of electrospun Vectran nanofiber mats after removal of PEO.
}

\section{Introduction}

Liquid crystalline polymers (LCPs) represent a class of polymers that are well known for their excellent mechanical properties, thermal and chemical resistance, and low density, which result in exceptional specific properties. ${ }^{1}$ Unlike conventional polymers, they crystallize from an ordered and oriented molecular phase intermediate between an isotropic liquid phase and a crystalline solid, or amorphous glassy phase.

Ordered liquid crystalline phases are mainly classified as nematic, cholesteric and smectic. The structural basis for each of them is a state of matter where the degree of molecular order is intermediate between the perfect three-dimensional, longrange positional and orientational order found in solid crystals, and the mesomorphic state with the absence of a long-range order, as found in isotropic liquids, gases and amorphous solids. ${ }^{2-4}$ The nematic liquid crystal, $\mathrm{N}$ phase, possesses a longrange orientational order but only a short-range positional order. Nematics are the most important members of the liquid crystal family and are widely used in the display industry.,5,6 The cholesteric phase is similar to the nematic phase on a local scale. Like the nematic phase, it can be described by a molecular alignment with respect to a direction towards which all the molecular orientations will be biased, at least locally (namely, a director). However, the direction in the cholesteric phase is twisted about an axis normal to the molecular orientation, following a helical path. ${ }^{3,5,6}$ Smectic phases are characterized by

${ }^{a}$ Department of Materials Engineering and Industrial Technologies, University of Trento, Via Mesiano 77, 38123 Trento, TN, Italy

${ }^{b}$ Department of Mechanical and Industrial Engineering, University of Illinois at Chicago, 842 W. Taylor St, 60607-7022 Chicago, IL, USA. E-mail: ayarin@uic.edu layered structures, with additional order being possible in each layer. Within the layers, the centers of molecules are arranged in equidistant planes. The planes are allowed to move perpendicular to the layers, and, within the layers, different arrangements of molecules are possible. The modifications of the smectic phase are labeled according to the arrangement of the molecules within the layers. ${ }^{3,5,6}$ There are also other liquid crystalline phases (e.g. cubic, hexagonal, lamellar, columnar). Since they are not frequently present in thermotropic liquid crystals, they will not be detailed in this study. ${ }^{4}$

Among the main uses of LCPs, the following applications should be mentioned: production of high precision moldings for use in the electronics industry, multi-way electrical connectors, components of printers and disk drives, transformer bobbins and encapsulation for surface-mounted silicon chips. $^{3}$ LCPs can be broadly classified into three classes: (a) aromatic polyamides, (b) aromatic heterocycles, and (c) aromatic copolyesters. ${ }^{1}$ Aromatic polyamide fibers, commonly known as aramid fibers, are obtained from polyamides containing aromatic rings along the main chain, and the most known commercial products are Kevlar ${ }^{\circledR}$ and Twaron ${ }^{\circledR}$. Aromatic heterocyclic polymers are lyotropic materials and are characterized by wholly aromatic molecular structures with fused heterocyclic rings along the main chain; PBI and Zylon ${ }^{\circledR}$ are two examples in this category. Aromatic copolyester polymers possess thermotropic behavior and are characterized by a molecular structure with a high degree of linearity and rigidity that allow formation of ordered phases over a wide temperature range; among them, three examples are Vectra ${ }^{\circledR}, \mathrm{Xydar}^{\circledR}$ and Ekonol ${ }^{\circledR}{ }^{1}$ The melt-spun Vectra-based fiber, commercially known as Vectran, is superior to aramid fibers in several ways: it is highly resistant to creep, it resists flex or fold fatigue 
and abrasion, and it has better long-term resistance to UV degradation., ${ }^{3,5}$

When diameters of polymer fibers are reduced from micrometers to a few hundred nanometers, several attractive characteristics may be induced. ${ }^{7}$ Some of the characteristics are - a very large surface area to volume ratio (this ratio for nanofibers can be $10^{3}$ times larger than that of microfibers), flexibility in surface functionalities, change in the crystalline structure, ${ }^{8}$ and superior mechanical performances (e.g. stiffness and tensile strength). ${ }^{7}$ There are several previous studies regarding nanofibers forming from LCPs [e.g. aramid, BB5(3-Me) and liquid crystal polysiloxane with cholesterol]. ${ }^{9-13}$ On the other hand, to the best of our knowledge, there have been no reports on the formation of Vectran nanofibers. Electrospinning ${ }^{12,14-16}$ seems to be one of the best possible routes to form such nanofibers. In the electrospinning process, an electric field of strength about $1 \mathrm{kV} \mathrm{cm}^{-1}$ is applied to a needle through which polymer solution is delivered. When the applied electric field overcomes surface tension and the viscoelastic forces in the droplet pendent or sessile at the needle exit, a charged jet of the polymer solution is ejected. The jet undergoes the electricallydriven bending instability which stretches it dramatically, while the solvent evaporates. ${ }^{16}$ As a result, solidified nanofibers are formed and are deposited on a solid collector, which is a grounded counter-electrode. Unlike conventional spinning methods, where the smallest diameters of tens of micrometers can be achieved, the electrospinning technique can easily produce fibers with diameters from $\sim 10 \mathrm{~nm}$ to $1 \mu \mathrm{m} .{ }^{12,14-16}$

Electrospinning of liquid crystalline aromatic polyamide poly(p-phenylene terephthalamide) (PPTA), commercialized under the trade name Kevlar $49{ }^{\circledR}$ (Dupont), was demonstrated in the seminal work of Reneker's group. ${ }^{12}$ They collected nanofibers on the free surface of water in a grounded bath where PPTA solution in sulfuric acid precipitated, and solidified fibers were formed. Acetoxypropyl cellulose (APC), a lyotropic cholesteric LCP, was successfully electrospun as microfibers $(\sim 2.75 \mu \mathrm{m})$ using a standard electrospinning setup with a solid counter-electrode collector. ${ }^{17}$ Liquid crystal elastomers (LCE) were electrospun as microfibers with an average diameter of $1.5 \mu \mathrm{m}$ from a solution of photocross-linkable polymer using a conventional electrospinning apparatus. ${ }^{13}$ Only beaded fibers were obtained, and cross-linking was conducted using UV light as a post-processing step. Also, the relationship between the diameter of electrospun LCP fibers [BB-5(3-Me)] and their internal structure and molecular orientation was studied. ${ }^{10}$

The aim of this work is to electrospin liquid crystalline polyester (Vectran) or its blends with PEO as nanofibers, and characterize their crystalline and mechanical properties prior to, and after the removal of PEO. Vectran nanofibers would open an avenue for applications of this popular liquid crystalline polymer in ultra-strong composites. It is emphasized that due to the above-mentioned reasons, none of the previously reported LCP nanofibers and/or microfibers can compete with Vectran in achieving ultra-strong nanofibers.

In Section 2 the materials and methods to produce Vectran nanofibers are described, as well as the instrumentation used and the thermal treatment procedure are detailed. In subsequent Section 3 the main properties of the resulting nanofibers are analyzed and discussed. Also, the influence of the optimized thermal treatment on the nanofibers is investigated using various characterization methods, and their tensile strength is tested. In Section 4 the conclusions are drawn.

\section{Experimental}

\subsection{Materials}

Kuraray supplied the as-spun Vectran LCP yarn used in this work under the trade name of Vectran NT having a linear density of 750 denier and 150 filaments per yarn. Vectran is a copolymer of 4-hydroxybenzoic acid (HBA) and 2-hydroxy6-naphtonic acid (HNA) with a molar ratio of $73 / 27$, respectively. ${ }^{18,19}$ Vectran NT has a molar mass of 290.27 Da and a molecular weight higher than $20 \mathrm{kDa}^{20}$ The fibers possess an almost circular cross-section with an average diameter of $25.5 \pm$ $2.1 \mu \mathrm{m} .{ }^{21}$ Poly(ethylene oxide) (PEO, molar mass $44.05 \mathrm{Da}, M_{\mathrm{w}}=$ $600 \mathrm{kDa}$ ), chloroform (>99.8\%) and pentafluorphenol (PFP, $>98 \%$ ) were purchased from Sigma-Aldrich. All materials were used as received without any further purification.

\subsection{Sample preparation}

Vectran solution (1 wt\%) was prepared by dissolving $0.2 \mathrm{~g}$ of LCP in $20 \mathrm{~g}$ of a mixture of two solvents, chloroform and pentafluorphenol, with a 70/30 ratio by weight, respectively. Stirring for $10 \mathrm{~h}$ at room temperature resulted in a yellow-clear solution. PEO solution (1.85 wt\%) was prepared by dissolving $0.37 \mathrm{~g}$ of PEO in $19.63 \mathrm{~g}$ of chloroform under magnetic stirring for $5 \mathrm{~h}$. Then, the Vectran solution was mixed with the PEO solution at a ratio of $15: 1$ by weight, respectively. The as-spun nanofibers produced in the present work possess the Vectran-PEO weight ratio of $8.19: 1$, correspondingly.

\subsection{Electrospinning process}

To electrospin Vectran or its blends with PEO, a conventional electrospinning setup was used, with aluminum foil used as a collector. ${ }^{22}$ The controlled power generator employed was a Glassman High Voltage model $\mathrm{EH}$, that can generate a DC voltage in the range $0-30 \mathrm{kV}$. A pump NE-1000 from New Era Pump Systems, Inc. was used to ensure a continuous supply of polymer solution during the process. The voltage was set at $10 \mathrm{kV}$ at a solution flow rate of $1.5 \mathrm{~mL} \mathrm{~h}^{-1}$, and the distance between the collector and the needle was kept equal to $10 \mathrm{~cm}$. The samples obtained were dried for $24 \mathrm{~h}$ at room temperature.

\subsection{Heat treatment}

In the past two decades annealing on liquid crystalline polymers was widely studied, but never attempted on LCP nanofibers. The annealing consisted of increasing the temperature of the nanofibers close to the melting temperature, and keeping them under these conditions for a pre-determined amount of time. The annealing used in the present work resembles that for commercial Vectran microfibers. ${ }^{23-27}$ The annealing was used to enhance the mechanical properties of Vectran, as well as its melting temperature. The thermal treatment was performed in 
an oven in air in the temperature range $250-300{ }^{\circ} \mathrm{C}$, and the treatment duration was in the range $15-24 \mathrm{~h}$.

\subsection{Thermal analysis}

Differential scanning calorimetry (DSC) measurements were performed using a Mettler DSC 30 Low Temperature Cell and a Mettler TC $15 \mathrm{TA}$ Controller. The heating rate was $10^{\circ} \mathrm{C} \mathrm{min}^{-1}$ under a nitrogen flux of $100 \mathrm{~mL} \mathrm{~min}{ }^{-1}$.

Thermogravimetric analysis (TGA) was applied, and measurements were performed using a modulated TGA Q5000IR by TA Instrument. The heating rate was $10{ }^{\circ} \mathrm{C} \mathrm{min}-1$ under a nitrogen flux of $25 \mathrm{~mL} \mathrm{~min}^{-1}$.

\subsection{Mechanical analysis}

Electrospun nanofiber mats were cut into rectangular specimens measuring $4 \times 30 \mathrm{~mm}^{2}$ and mounted on window-like holders with a gage length of $20 \mathrm{~mm}$. Tensile tests were conducted on 15 as-spun mat specimens. Also a sample (consisting of 13 heat-treated specimens) was subjected to the tensile test. The tensile tests were conducted using a universal testing machine (Instron, model 4502) with a $10 \mathrm{~N}$ load cell (Instron, model $2518-808$, with a nominal accuracy of $0.025 \%$ of the load cell full scale) at a cross-head speed of $5 \mathrm{~mm} \mathrm{~min}^{-1}$.

\subsection{Observations}

Scanning electron microscopy (SEM) images were obtained using JEOL JSM-6320F and Supra 40 Zeiss microscopes under high vacuum and secondary electron detector operating mode. Transmission electron microscopy (TEM) was performed using a JEOL JEM-3010 microscope. Optical observations were done using an Olympus BX51 microscope in refraction mode.

The selected area electron diffraction (SAED) method is similar to X-ray diffraction but uses electrons rather than X-rays. Because of that, the examined region can be as small as a few nanometers. SAED images are obtained by using an aperture in the virtual image plane of the microscope to select a region of interest such as an individual nanofiber. Only the electrons falling inside the dimensions of the aperture will be analyzed. The resulting scattered electrons are then imaged in the diffraction mode of the microscope. The circular pattern that appears is in essence a two-dimensional scattering pattern. This two-dimensional nature gives extra information about the orientation of the lattice scattering planes compared to a onedimensional X-ray diffraction pattern. ${ }^{28}$

\section{Results and discussion}

Prior to trials of electrospinning of Vectran and PEO blends, electrospinning of pure Vectran solution was attempted. The results are shown in the SEM images of Fig. 1. It is seen that using electrospinning of pure Vectran solution, continuous and uniform nanofibers could not be achieved. This result stems from different factors. The rigid molecular structure of Vectran results in a limited viscoelastic behavior, which is the primary condition for spinnability in electrospinning ${ }^{14-16}$ The situation is worsened because of the tendency of LCP to form aggregates
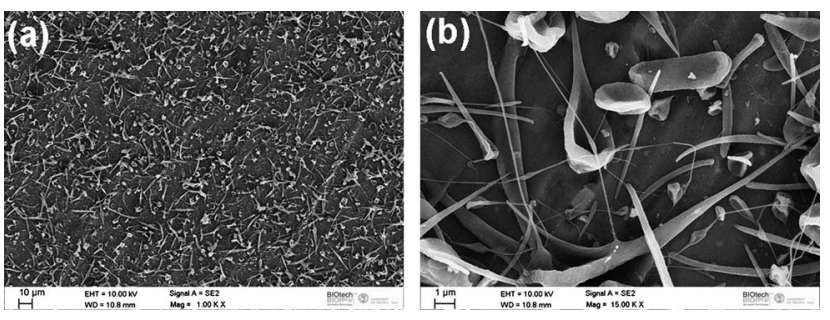

Fig. 1 (a) and (b) SEM images of the electrospun Vectran (1 wt\%) at different magnifications.

after a fast evaporation of one of the solvents present in the Vectran solution (chloroform). ${ }^{9}$ The insufficient spinnability results in rupture of electrospun Vectran jet producing a mix of dried beads and non-continuous fibers of varying diameters and shapes ( $c f$. Fig. 1). Several different Vectran solutions with concentrations in the range 0.5 to $2 \mathrm{wt} \%$ have been used in these experiments, as well as different ratios of chloroform/PFP were attempted. However, in all cases only results similar to those shown in Fig. 1 were observed.

The use of spinnable additives, a procedure known as the host-guest approach, ${ }^{29}$ became necessary in order to achieve good quality Vectran-based nanofibers. PEO was chosen as a "host", since it is a flexible high-molecular-weight polymer widely used to enhance viscoelasticity and spinnability of the solution. Moreover, PEO is also soluble in chloroform, one of the solvents used in the Vectran solution. Due to its high molecular weight, a sufficient spinnability was achieved even at relatively small concentrations of PEO (1.85 wt\%). This facilitates the subsequent removal of PEO from nanofibers without changing their morphology as shown in the next sections.

The PEO solution was added to the Vectran solution in different ratios, with $1: 15$ being the most effective, and the resulting nanofibers had a Vectran-PEO ratio of $8.19: 1$. In Fig. 2(a) continuous nanofibers electrospun from the PEOVectran blend are shown. Their average diameter is $195 \mathrm{~nm}$ and the molar ratio of Vectran-PEO is 1.25.

After electrospinning of PEO-Vectran solutions, the resulting nanofiber mats were dried to evaporate chloroform completely. After that, only PFP, PEO and Vectran remain in the fibers. In previous works dealing with Vectran solutions, dichloromethane (DCM) was used to extract PFP from the film produced. ${ }^{30}$ Due to the presence of PEO, DCM could not be used in the present work.

In order to remove PEO after fiber formation, the nanofiber mats were immersed for $24 \mathrm{~h}$ into a bath containing ethanol and water ( $c f$. SEM images in Fig. 2). In Fig. 2(b) the Vectran fibers seem to be swollen in comparison to Fig. 2(a), partly due to the expected dissolution of PEO after the immersion into the water-ethanol bath, and partly due to the plasticizing effect stemming from water absorption. The latter means that water remains entrapped between the Vectran and the remnant PEO macromolecules in the nanofibers causing an increase in the fiber diameter. Despite the reduction of the PEO content in the nanofibers, the immersion into the water-ethanol bath does not completely eliminate PEO from the nanofibers. 

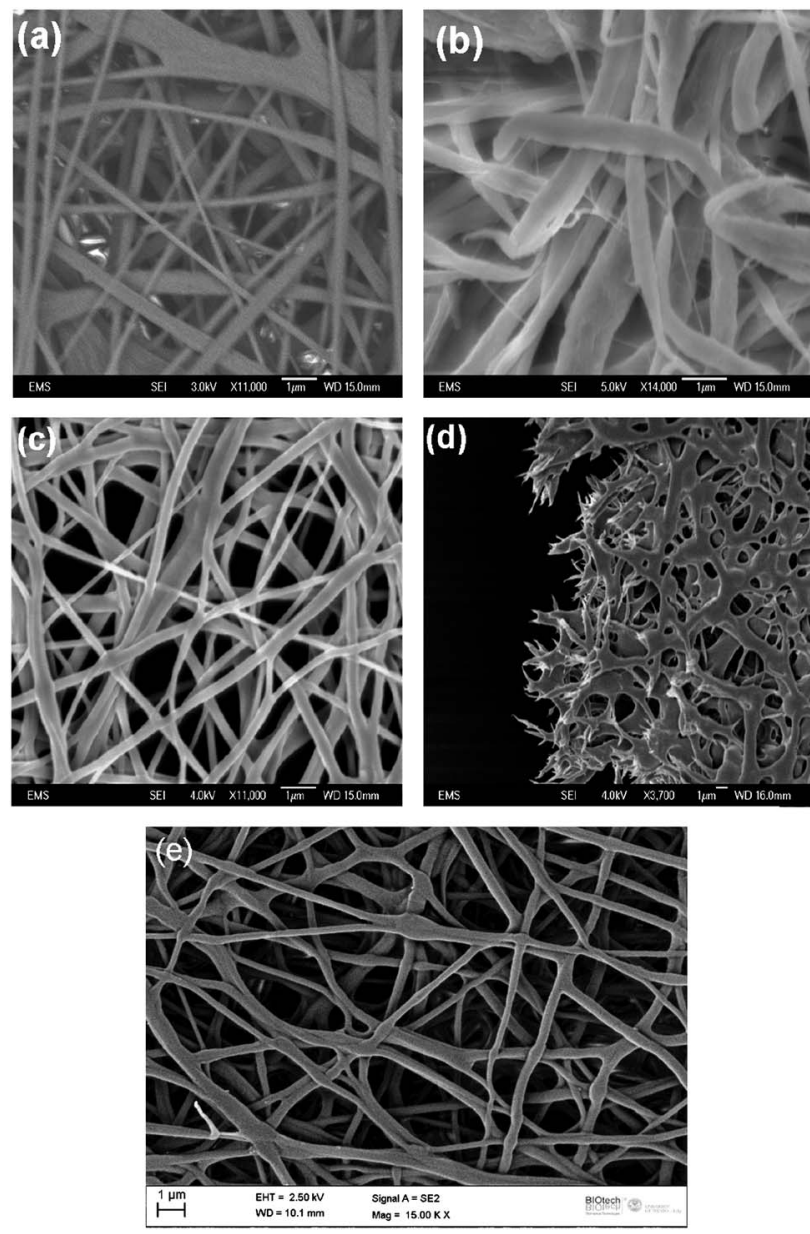

Fig. 2 SEM images. (a) Electrospun Vectran-PEO mat. (b) Electrospun VectranPEO mat after the immersion into a water-ethanol bath to dissolve PEO. (c) Heattreated electrospun Vectran-PEO fibers. (d) Electrospun Vectran-PEO mat after immersion into a water-ethanol bath and subsequent heat-treatment. (e) Electrospun Vectran-PEO mat after heat treatment and subsequent immersion into a water-ethanol bath.

Therefore, instead of using a solvent-based PEO removal procedure, a heat treatment was used to ensure complete removal of PEO from nanofibers, as well as to enhance their thermal stability and mechanical properties. In Fig. 2(c) the heat-treated nanofibers, which were not immersed into the water-ethanol bath, are seen to be continuous and uniform. On the other hand, in Fig. 2(d) the nanofibers, which were heat treated after the immersion into the water-ethanol bath, look conglutinated. Fig. 2(b) demonstrates that the fiber mat swells after the immersion into the water-ethanol bath, and then, perhaps, partially melts during heat treatment. As a result, nanofibers are additionally sintered, with the average diameter increasing to about $1 \mu \mathrm{m}$.

To demonstrate that swelling was due to the presence of PEO and not related to Vectran itself, the same immersion procedure was applied to electrospun mats after heat treatment. Fig. 2(e) shows that, indeed, no swelling is visible in the heat-treated Vectran nanofiber mat.

\subsection{Thermal analysis}

To check whether the heat treatment resulted in a complete removal of PEO or not, both TGA and DSC techniques have been adopted. The partial dissolution of PEO present in the nanofibers after the immersion into a water-ethanol bath was also confirmed by TGA analysis. Degradation of PEO used in this work is expected in the range from $210{ }^{\circ} \mathrm{C}$ to $410{ }^{\circ} \mathrm{C} .{ }^{31}$ TGA curves reported in Fig. 3 show that the non-treated nanofibers that were not immersed into the water-ethanol bath lose $14.56 \%$ of their weight at $410{ }^{\circ} \mathrm{C}$, while non-treated nanofibers immersed into the bath lose $6.76 \%$ in weight at the same temperature. Also, the heat-treated nanofibers that were immersed into the water-ethanol bath lose $1.46 \%$, and the heattreated nanofibers that were not immersed into the bath lose $1.38 \%$. This slight difference in the weight loss could be explained by the different morphologies of the two mats, namely, a conglutinated morphology after the immersion and non-conglutinated nanofibers without immersion. Conglutination results in different surface areas and different amounts of energy required for the Vectran degradation. The commercial Vectran NT shows a higher weight loss (3.31\%) in comparison with the treated mats, as the results in Fig. 3 show. This higher loss is probably related to the size effect of the commercial fibers, and also to the lower thermal stability of Vectran NT without the annealing treatment. Our results confirm that the immersion alone is not enough to completely remove PEO from LCP nanofibers. However, after the heat treatment there are no traces of residual PEO in the nanofibers. It can be seen that immersion followed by heat treatment resulted in more conglutinated fibers with a larger diameter, and requires additional treatment steps. That is why the immersion stage was completely excluded and the nanofibers of the Vectran-PEO blend were only heat treated to eliminate PEO.

Vectran begins loosing weight at $460{ }^{\circ} \mathrm{C}$ when the first vibrations of the aromatic rings occur. These vibrations continue approximately up to $500{ }^{\circ} \mathrm{C}$, which is about $40{ }^{\circ} \mathrm{C}$

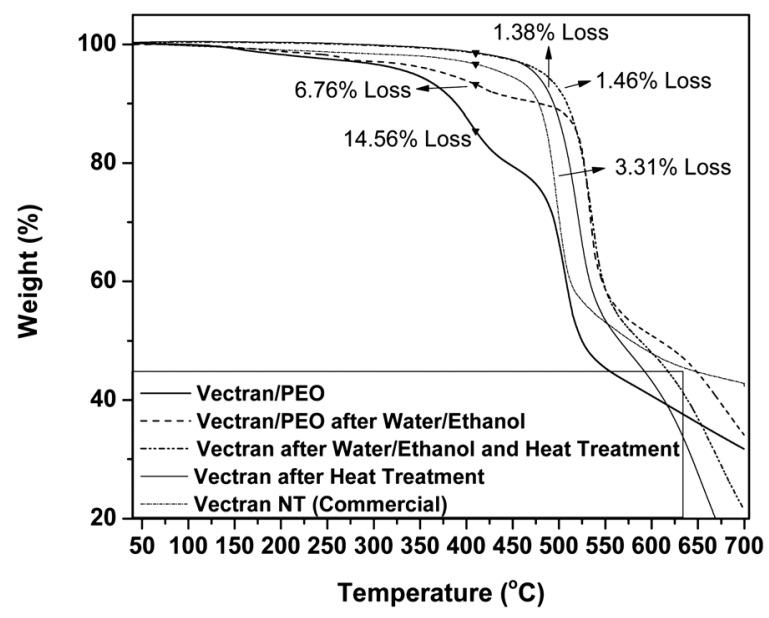

Fig. 3 Comparison of TGA data of the Vectran-PEO mat, Vectran-PEO mat after immersion into a water-ethanol bath, Vectran-PEO mat after heat treatment, and Vectran NT (commercial). 
below the temperature of the maximum weight loss of Vectran. From $510{ }^{\circ} \mathrm{C}$ onward there are $\mathrm{C}-\mathrm{O}, \mathrm{O}-\mathrm{H}$ and $\mathrm{C}-\mathrm{H}$ vibrations causing the actual degradation of Vectran. According to the existing scientific literature, the total degradation of Vectran completes at about $800{ }^{\circ} \mathrm{C} .{ }^{6}$

Even though TGA analysis conclusively proves a complete removal of PEO from the blended fibers, a question that still remains is how the heat treatment has influenced the crystalline structure of the electrospun composite nanofibers. The untreated commercially available fibers show two wide endothermic peaks $\left(T p_{1}, T p_{2}\right)$ [Fig. 4(a)] in DSC, which are attributed in the literature to an orthorhombic to nematic transition. ${ }^{\mathbf{1 8 , 2 3 - 2 5}}$ After heat treatment of commercial fibers, a new endothermic peak $\left(T m_{1}\right)$ appears, which overlaps with the two endothermic peaks $\left(T p_{1}, T p_{2}\right)$ present in the untreated fibers, and becomes a new melting temperature for such fibers. ${ }^{18,23,27}$ As reported in the previous studies, $T m_{1}$ could be related to the occurrence of inter-chain trans-esterification reactions. ${ }^{18,23,27}$

Note that initially the annealing of nanofibers was performed in air at $300{ }^{\circ} \mathrm{C}$ for $15 \mathrm{~h}$ to mimic a route previously used to anneal Vectran microfibers. ${ }^{27}$ On a visual inspection, nanofibers showed a typical color change. However, the observations

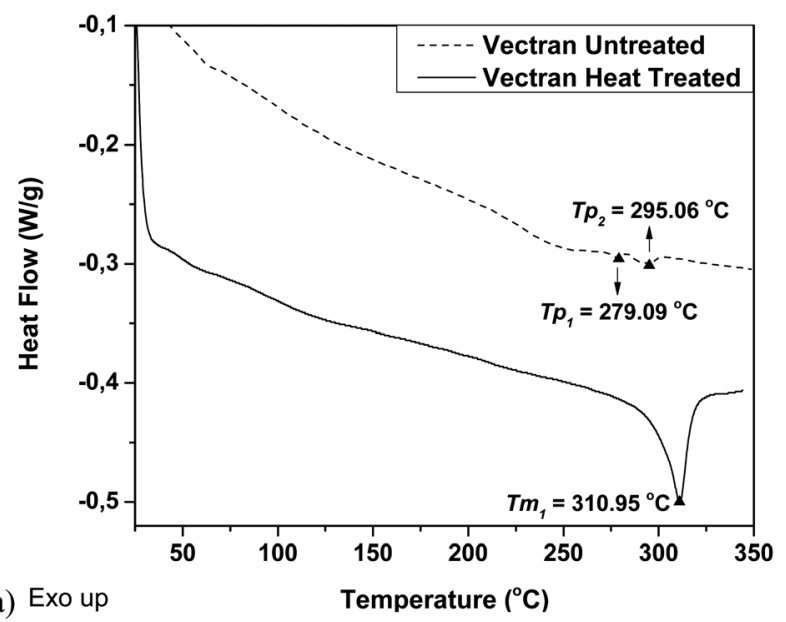

(a) Exo up

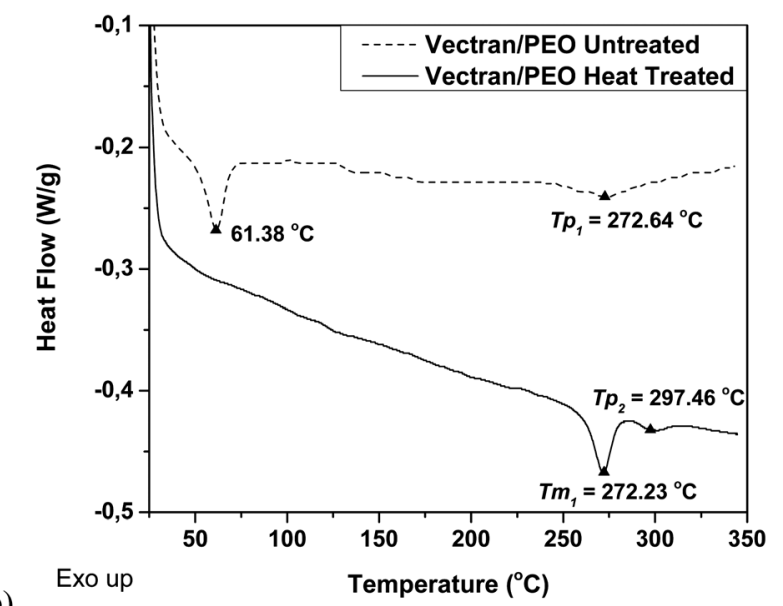

(b)

Fig. 4 DSC traces comparing (a) commercial Vectran microfibers before and after heat treatment, and (b) Vectran nanofibers before and after heat treatment. with an optical microscope revealed that the nanofiber mat has conglutinated and lost its fibrillar structure. This can be attributed to the fact that due to the smaller diameter of nanofibers, they have a higher surface area/volume ratio in comparison with microfibers. As a result, they melt at a temperature lower than the macro- and microscopic samples of the same polymer, as it was previously demonstrated with other nanofibers. ${ }^{26}$ In order to avoid nanofiber melting and conglutination, the heat treatment temperature was reduced and its duration increased. This annealing procedure was conducted at $250{ }^{\circ} \mathrm{C}$ during $24 \mathrm{~h}$ in air. The nanofibers showed the expected color change, whereas the optical microscopy observations did not reveal any loss in their fibrillar structure.

In Fig. 4(b) DSC traces of Vectran-PEO electrospun nanofibers are shown for two cases, before and after heat treatment. The DSC traces of the untreated Vectran nanofibers show an endothermic peak at $61.4{ }^{\circ} \mathrm{C}$. This peak is related to the melting point of PEO $\left(66^{\circ} \mathrm{C}\right),{ }^{31}$ confirming once again its presence in nanofibers before heat treatment. The $T p_{1}$ peak found in the untreated microfibers is significantly smoothened out, and the $\mathrm{Tp}_{2}$ peak has disappeared. These peaks are related to a change in the crystallographic phase of Vectran, and their smoothening could be explained by the presence of PEO, which does not allow the orthorhombic to nematic transitions in the LCP.

After the heat treatment, the peak known as $T m_{1}$ appears in the DSC traces of the nanofibers. Also, one of the two endothermic peaks $\left(T p_{2}\right)$ characteristic of Vectran microfibers appears, indicating the presence of a crystalline structure and a possible orthorhombic to nematic transition. The peak related to PEO is not present anymore, which confirms once again its complete elimination from the nanofibers.

It is emphasized that the $T m_{1}$ peak does not overlap with the two endothermic peaks as it happens in the commercially available microfibers subjected to a similar heat treatment [Fig. 4(a)]. The temperature corresponding to the $\mathrm{Tm}_{1}$ peak in the nanofibers is $272.2{ }^{\circ} \mathrm{C}$, which is lower by $38.7^{\circ} \mathrm{C}$ than the corresponding peak in the microfibers. According to the previous studies, this phenomenon could be explained mainly by the following three factors: (i) a higher surface area/volume ratio in comparison with microfibers; (ii) the plasticizing effect due to a residual solvent; and (iii) modification of the crystalline structure as a result of rapid solidification of polymer solutions in electrospinning. ${ }^{26}$ The hypothesis of residual solvent can be discarded since TGA and DSC analyses did not show any traces of it. Vectran is a liquid crystalline polymer, which means that it does not loose its crystalline structure in the liquid phase. From the SAED pattern, to be shown and discussed later, it could be seen that the presence of PEO, causes the loss of the Vectran crystalline structure before heat treatment. It will also be shown that although after the heat treatment the nanofibers demonstrate an increase in their crystalline orientation, their crystalline structure is still not absolutely identical to the commercial Vectran fibers. The latter possess a higher order in their crystalline structure due to the production process. Therefore, the modification of the crystalline structure due to the fast evaporation of solvents stems from the higher surface area/ volume ratio in nanofibers in comparison with microfibers. 

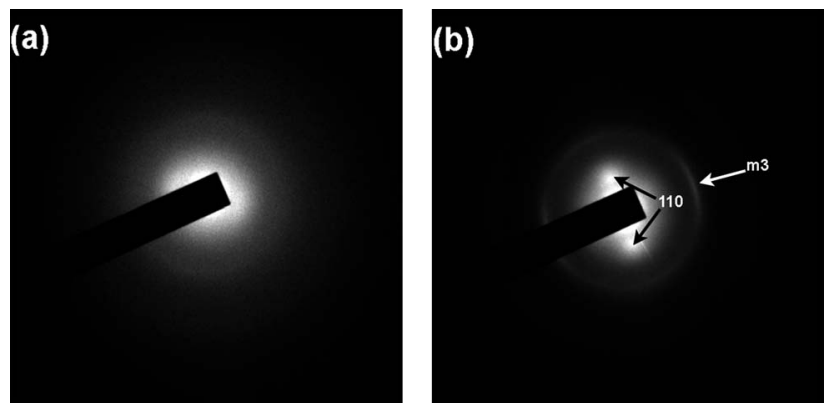

Fig. 5 SAED pattern for (a) Vectran nanofibers before, and (b) after heat treatment. The camera distance was $40 \mathrm{~cm}$ in both cases.

That increases the propensity to heat dispersion in the nanofibers and could explain the lower temperature of the $\mathrm{Tm}_{1}$ peak in comparison with the position of the same peak in the heattreated commercial fibers.

Although the thermal analysis clearly indicates the change in the crystalline order, the final clarification can only be obtained through the experimental evaluation of $d$-spacing in the nanofibers. It should be mentioned that instead of wide-angle X-ray scattering (WAXD) on nanofiber mats, selected area electron diffraction (SAED) on a single nanofiber was preferred. It was done with the goal to check whether SAED of any arbitrary nanofiber at any location reproduces the same $d$-spacing, as that of annealed Vectran. ${ }^{32-35}$ Only then it could be concluded that every single nanofiber underwent the lateral packing of crystalline structure. SAED was performed on both the untreated and heat-treated Vectran nanofibers, and representative images are shown in Fig. 5. It can be seen from Fig. 5(a) that the SAED pattern of an untreated nanofiber does not show any sharp ring. The presence of a diffused halo clearly indicates that the existence of PEO had hampered lateral packing thus yielding an amorphous structure. Otherwise, meridional and equatorial reflections would be visible. ${ }^{32-35}$

In Fig. 5(b) the SAED patterns show a strong meridional reflection and one strong equatorial reflection. From the calculation of the $d$-spacing it was found that the $d$-spacing corresponding to the meridional and equatorial reflections was $2.2 \AA$ and $4.7 \AA$ A respectively. From ref. 25 it can be found that in Vectran there should be three meridional reflection at the $d$-spacings $\sim 6.73(\mathrm{~m} 1), 3.06$ (m2) and 2.07 (m3) A. However, as ref. 25 shows, the intensity of $\mathrm{m} 1$ and $\mathrm{m} 2$ is quite low and if they were visible in the SAED pattern, they would have been near the center. The electron beam is so bright that in spite of using a beam stopper, the CCD camera was completely blinded near the center, which makes observation of $\mathrm{m} 2$ and $\mathrm{m} 1$ impossible. The $d$-spacing value clearly shows that the meridional reflection corresponds to $\mathrm{m} 3 .^{31}$ Besides the $d$-spacing, the equatorial reflection also shows that it corresponds to the 110 plane. ${ }^{31}$ The latter, in conjunction with the thermal analysis, conclusively points out that annealing not only destroyed PEO but also improved the lateral order of the random crystal orientation. Also, the random sequences were crystallized into ordered crystals with higher melting temperatures. The increase in the thermal stability after annealing is strongly related to the
Table 1 Tensile properties of heat-treated and untreated Vectran nanofiber mats

\begin{tabular}{lcc}
\hline Property & $\begin{array}{l}\text { Vectran-PEO } \\
\text { untreated }\end{array}$ & $\begin{array}{l}\text { Vectran-PEO } \\
\text { heat-treated }\end{array}$ \\
\hline $\begin{array}{l}\text { Tensile modulus } \\
{[\mathrm{MPa}]}\end{array}$ & $29.5 \pm 15.4$ & $29.8 \pm 10.0$ \\
$\begin{array}{l}\text { Tensile strength } \\
{[\mathrm{MPa}]}\end{array}$ & $0.96 \pm 0.34$ & $4.17 \pm 2.17$ \\
$\begin{array}{l}\text { Elongation at } \\
\text { break [\%] }\end{array}$ & $3.01 \pm 0.80$ & $7.77 \pm 2.43$ \\
\end{tabular}

enhancement of structural order. ${ }^{35}$ The existence of an almost circular $\mathrm{m} 3$ ring (with a mix of bright and fading parts) in Fig. 5(b) instead of the arches seen in ref. 25, clearly shows that in our case the crystallites responsible for the $\mathrm{m} 3 \mathrm{ring}$ are not absolutely aligned with the nanofiber axis. However, it can be seen from Fig. 5 that $\mathrm{m} 3$ is brightest in the longitudinal direction of the nanofibers, and the bright 110 plane is almost at the right angle to it, which appears to be the preferential direction of the crystallite orientation.

\subsection{Mechanical analysis}

Heat treatment is intended not only to remove residual PEO and improve thermal stability of Vectran, but also to increase mechanical properties of Vectran nanofibers. According to the previous studies, the mechanical properties of Vectran microfibers are markedly different before and after heat treatment. ${ }^{23,27,36}$ Our data in Table 1 show that the heat treatment of Vectran nanofiber mats increased tensile strength by $334 \%$ and the elongation at break by $158 \%$. On the other hand, the tensile modulus of the mats does not change significantly, which is similar to the results obtained after annealing of Vectran microfibers. ${ }^{23,27,36}$

The improvement in the tensile properties of Vectran after a heat treatment has generally been attributed to an increase in the molecular weight. ${ }^{23,24}$

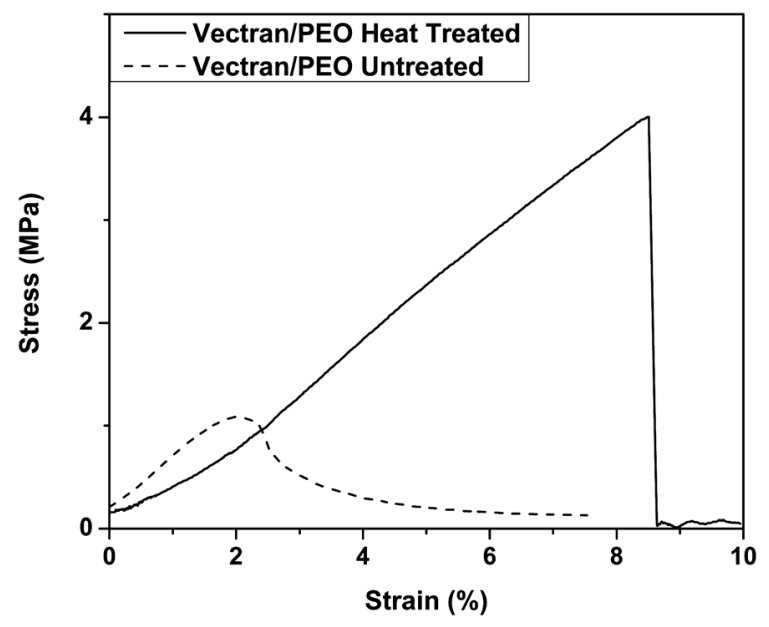

Fig. 6 Representative stress-strain curves of heat-treated and untreated Vectran-PEO nanofiber mats. 
It is emphasized that PEO initially present in the nanofibers before the heat treatment acts as a plasticizer and results in ductile behavior of the untreated mats (Fig. 6). However, after the annealing when PEO has been completely removed, the nanofiber mats become much stiffer (Fig. 6), which was the main goal of this work. Although, the mat strength is smaller than that for individual heat-treated Vectran microfibers $[1.52 \pm 0.12 \mathrm{GPa}],{ }^{27}$ one should keep in mind that tensile strength of nanofiber mats is always much lower than that of an individual nanofiber. ${ }^{14,37}$ The main reasons are that (i) nanofibers in electrospun mats are randomly oriented and cannot be deformed only along their main axis as is commonly done when single microfibers are tested; (ii) the real crosssection of electrospun mats is difficult to be assessed and an average value is generally considered by measuring the external dimensions with a caliper; (iii) while measuring tensile strength of the nanofiber mats, we also inevitably measure effective strength of the inter-fiber bonds, since in the electrospun mats fibers are ill-entangled; (iv) electrospun nanofibers can possess significant porosity due to solvent evaporation and PEO removal. Overall, the factors listed as (i) to (iv) yield mats with a much lower tensile strength than individual nano- and microfibers.

\section{Conclusions}

Pure Vectran solutions possess insufficient spinnability to produce continuous and uniform nanofibers using electrospinning. The use of a host-guest approach with the host being a high molecular weight PEO was employed to improve spinnability of Vectran. Then, continuous and uniform nanofibers with an average diameter of $195 \mathrm{~nm}$ were formed.

In order to remove the remnant PEO, water-ethanol immersion was tried. It removed about $8 \%$ of the remaining PEO but led to nanofiber swelling. Therefore, the immersion stage was found to be inefficient and was avoided. Instead, PEO was eliminated by heat treatment. The heat treatment of Vectran microfibers was optimized at $250{ }^{\circ} \mathrm{C}$ for $24 \mathrm{~h}$ in air. TGA and DSC analyses confirmed the complete removal of the remaining PEO and solvents after heat treatment. Also, DSC showed that PEO changes the crystalline structure of as-spun Vectran-PEO nanofibers making them an almost amorphous material without crystalline orientation. However, after heat treatment an endothermic peak $\left(T p_{2}\right)$ related to a formed crystalline structure was observed using DSC. Furthermore, SAED confirmed the absence of an ordered crystalline structure before heat treatment and a low degree of crystallinity and a welloriented structure in the Vectran nanofibers after heat treatment.

Finally, the annealing of Vectran nanofiber mats increases thermal stability due to the enhancement of the structural order. Correspondingly, tensile tests show a significant improvement of the mechanical properties. The mechanical behavior in tensile tests changed from relatively soft to stiff, and the elongation at break has also increased after PEO was eliminated.

\section{References}

1 A. Pegoretti and M. Traina, in Handbook of Tensile Properties of Textile and Technical Fibres, ed. A. R. Bunsell, Woodhead Publishing Ltd, Cambridge, UK, 2009, pp. 354-436.

2 G. Friedel, Ann. Phys., 1922, 201.

3 A. M. Donald, A. H. Windle and S. Hanna, Liquid Crystalline Polymers, Cambridge University Press, 2006.

4 D. Demus, J. Goodby, G. W. Gray, H.-W. Spiess and V. Vill, Handbook of Liquid Crystals: Fundamentals, Wiley-VCH, 1998.

5 X. Wang, Q. F. Zhou and Q. Zhou, Liquid Crystalline Polymers, World Scientific Pub. Co., 2004.

6 T. S. Chung, Thermotropic Liquid Crystal Polymers: Thin-Film Polymerization, Characterization, Blends, and Applications, Technomic Publishing Company, 2001.

7 Z. M. Huang, Y. Z. Zhang, M. Kotaki and S. Ramakrishna, Compos. Sci. Technol., 2003, 63, 2223-2253.

8 J. S. Stephens, D. B. Chase and J. F. Rabolt, Macromolecules, 2004, 37, 877-881.

9 Y. G. Wu, Q. An, J. X. Yin, T. Hua, H. M. Xie, G. T. Li and H. Tang, Colloid Polym. Sci., 2008, 286, 897-905.

10 K. Nakashima, K. Tsuboi, H. Matsumoto, R. Ishige, M. Tokita, J. Watanabe and A. Tanioka, Macromol. Rapid Commun., 2010, 31, 1641-1645.

11 D. H. Reneker and I. Chun, Nanotechnology, 1996, 7, 216223.

12 G. Srinivasan and D. H. Reneker, Polym. Int., 1995, 36, 195201.

13 S. Krause, R. Dersch, J. H. Wendorff and H. Finkelmann, Macromol. Rapid Commun., 2007, 28, 2062-2068.

14 D. H. Reneker, A. L. Yarin, E. Zussman and H. Xu, Adv. Appl. Mech., 2007, 41, 43-195.

15 D. H. Reneker and A. L. Yarin, Polymer, 2008, 49, 23872425.

16 D. H. Reneker, A. L. Yarin, H. Fong and S. Koombhongse, J. Appl. Phys., 2000, 87, 4531-4547.

17 J. P. Canejo, J. P. Borges, M. H. Godinho, P. Brogueira, P. I. C. Teixeira and E. M. Terentjev, Adv. Mater., 2008, 20, 4821-4825.

18 J. D. Menczel, G. L. Collins and S. K. Saw, J. Therm. Anal., 1997, 49, 201-208.

19 H. N. Yoon, L. F. Charbonneau and G. W. Calundann, Adv. Mater., 1992, 4, 206-214.

20 X. Wang, J. Engel and C. Liu, J. Micromech. Microeng., 2003, 13, 628-633.

21 A. Pegoretti, A. Zanolli and C. Migliaresi, Compos. Sci. Technol., 2006, 66, 1953-1962.

22 A. L. Yarin, S. Koombhongse and D. H. Reneker, J. Appl. Phys., 2001, 89, 3018-3026.

23 E. Kalfon-Cohen, A. Pegoretti and G. Marom, Polymer, 2010, 51, 1033-1041.

24 J. Sarlin and P. Tormala, J. Appl. Polym. Sci., 1993, 50, 12251231.

25 J. Sarlin and P. Tormala, J. Polym. Sci., Part B: Polym. Phys., 1991, 29, 395-405. 
26 R. Srikar, T. Gambaryan-Roisman, C. Steffes, P. Stephan, C. Tropea and A. L. Yarin, Int. J. Heat Mass Transfer, 2009, 52, 5814-5826.

27 T. Medeiros Araujo and A. Pegoretti, in 15th European Conference on Composite Materials, ECCM, Venice, 2012.

28 C. van Gulijk, K. M. de Lathouder and R. Haswell, Carbon, 2006, 44, 2950-2956.

29 A. L. Yarin, E. Zussman, J. H. Wendorff and A. Greiner, J. Mater. Chem., 2007, 17, 2585-2599.

30 K. Yonetake, T. Sagiya, K. Koyama and T. Masuko, Macromolecules, 1992, 25, 1009-1010.

31 K. Saeed, M. Ishaq and M. Ilyas, Turk. J. Chem., 2011, 35, 237-243.
32 E. Kalfon-Cohen, G. Marom, A. Weinberg, E. Wachtel, C. Migliaresi and A. Pegoretti, Polym. Adv. Technol., 2007, 18, 771-779.

33 E. Kalfon-Cohen, G. Marom, E. Wachtel and A. Pegoretti, Polymer, 2009, 50, 1797-1804.

34 H. C. Langelaan and A. P. de Boer, Polymer, 1996, 37, 56675680.

35 A. Kaito, M. Kyotani and K. Nakayama, Macromolecules, 1990, 23, 1035-1040.

36 A. Pegoretti, A. Zanolli and C. Migliaresi, Compos. Sci. Technol., 2006, 66, 1970-1979.

37 E. Zussman, M. Burman, A. L. Yarin, R. Khalfin and Y. Cohen, J. Polym. Sci., Part B: Polym. Phys., 2006, 44, 1482-1489. 